\title{
Towards Atom Probe Tomography of Hybrid Organic-Inorganic Nanoparticles
}

\author{
Lyle M. Gordon, Michael J. Cohen and Derk Joester
}

Department of Materials Science and Engineering, Northwestern University, Evanston, IL 60208 USA

Surface functionalization of inorganic nanoparticles is widely used to modify material properties for a variety of applications including medical therapies and diagnostics. While inorganic nanoparticles may be characterized via electron microscopy and X-ray scattering, study of hybrid nanomaterials and, in particular, buried interfaces is very challenging. Here we discuss the application of atom probe tomography (APT) to imaging organic-inorganic interfaces of biological nanoparticles, ferritin, as a model system. Ferritin is a structurally well-characterized protein, critical across biology to store some 4500 iron atoms and transport iron as mineralized Fe (III) [1]. This 24-subunit $12 \mathrm{~nm}$ protein has a spherical $8 \mathrm{~nm}$ core into which $\mathrm{Fe}$ (II) enters and is subsequently oxidized.

APT is uniquely capable of providing the necessary chemical and structural insight at the atomic scale by directly probing the location and chemical identity of the atoms within a small sample of material. APT consists of a field-ion microscope coupled to a time-of-flight mass spectrometer, providing chemical and three-dimensional positional information of ions successively field evaporated from a sharp sample. The advent of ultraviolet laser pulsing has widely enlarged the scope of APT to include a range of insulating materials, including hybrid organic-inorganic materials [3]. APT is specifically adept at characterizing nanomaterials with internal organic-inorganic interfaces, due to its elemental sensitivity and spatial accuracy. We recently demonstrated this capability in biological minerals in the magnetite cusp of the chiton tooth [4] and vertebrate bone and dentin [5].

We report here on the characterization of native equine spleen ferritin using APT. Greene et al. recently used APT to analyse ferritin as a model for characterizing biological materials using APT. In this work, researchers used a solidified organic/salt matrix, which contained numerous ferritin complexes [6]. These APT tips of dense ferritin/salt deposits were able to undergo APT, providing a proof of concept through mass spectrometry results. However, they were unable to characterize individual proteins and their experiments suffered from drastic tip bending, preventing meaningful reconstructions [6]. To image individual ferritin protein complexes, we deposited a dilute solution of native equine spleen ferritin onto a silicon post coated with Au-Pd (Figure 1) [7]. Further encapsulation of the adsorbed molecules with Au-Pd and subsequent focused ion beam milling allowed for the fabrication of APT sample tips. This method allowed for a small number of ferritin complexes to be characterized. This work not only allows for resolution and characterization of ferritin, but also shows our ability to use APT to characterize discrete nanoparticles.

Atom probe tomography characterizes ferritin as spherical accumulations of iron and oxygen surrounded by carbon and nitrogen. Creating an isoconcentration surface we can identify the iron-rich core within the ferritin protein (Figure 2). A proximity histogram reveals the clear iron- and oxygen-rich core of ferritin, while the carbon concentration peaks just beyond core, corresponding with the organic protein shell. While APT does not enable designation between Fe (II) and Fe (III), we can see the accumulation and storage of iron, within a carbon and nitrogen rich sphere. This work shows the ability to characterize protein complexes and other organic nanoparticles in order to gain new insight to facilitate clinical and scientific advances. 
Figure 1. Left, Method for fabricating ferritin atom probe tomography sample tips. First, Au-Pd is sputter coated onto a silicon post, onto which a dilute ferritin solution is deposited, ferritin adsorbs to the post and excess salts are subsequently washed away. Au-Pd is then coated onto the sample to protect the ferritin from focused ion beam (FIB) damage and to create a matrix for the APT tip. FIB protocols are used to cut a sharp APT sample tip with encapsulated ferritin. Right, scanning electron micrograph of ferritin deposited onto an ion-beam sputtered polycrystalline Au-Pd substrate.

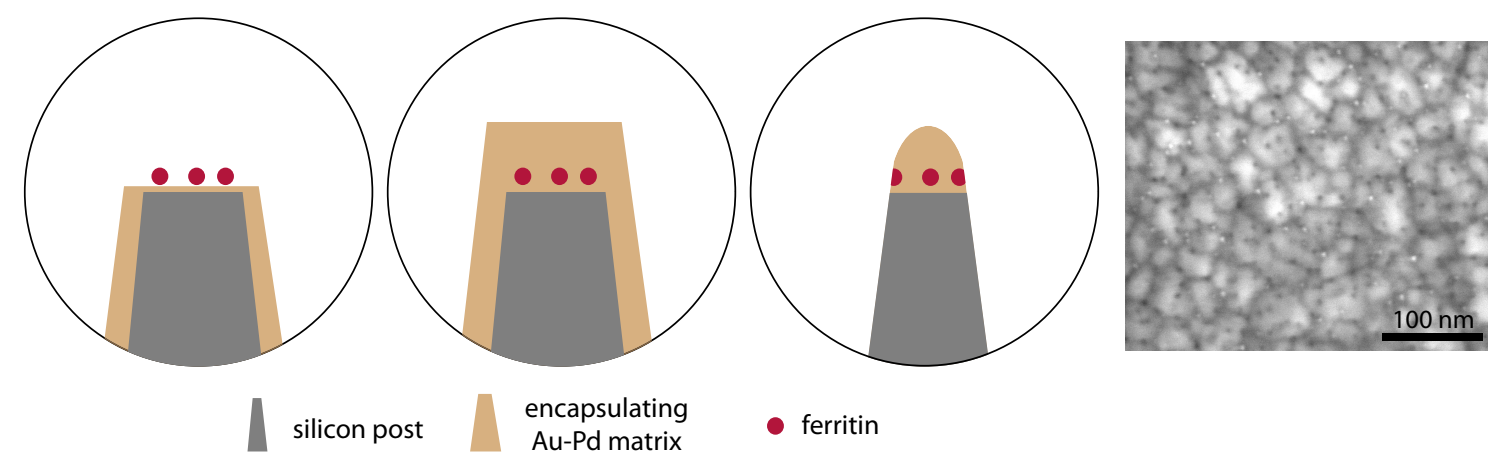

Figure 2. Left, APT reconstruction of single ferritin molecules including a five $\mathrm{Fe}$ ions $/ \mathrm{nm}^{3} \mathrm{Fe}$ ions $/ \mathrm{nm}^{3}$ isoconcentration surface. Right, proximity histogram, which plots the radial ionic concentration from the isoconcentration surface, showing atomic concentration of iron, carbon and oxygen relative to the interface between then core within the ferritin and the protein (defined as five Fe ions $\left./ \mathrm{nm}^{3}\right)$.
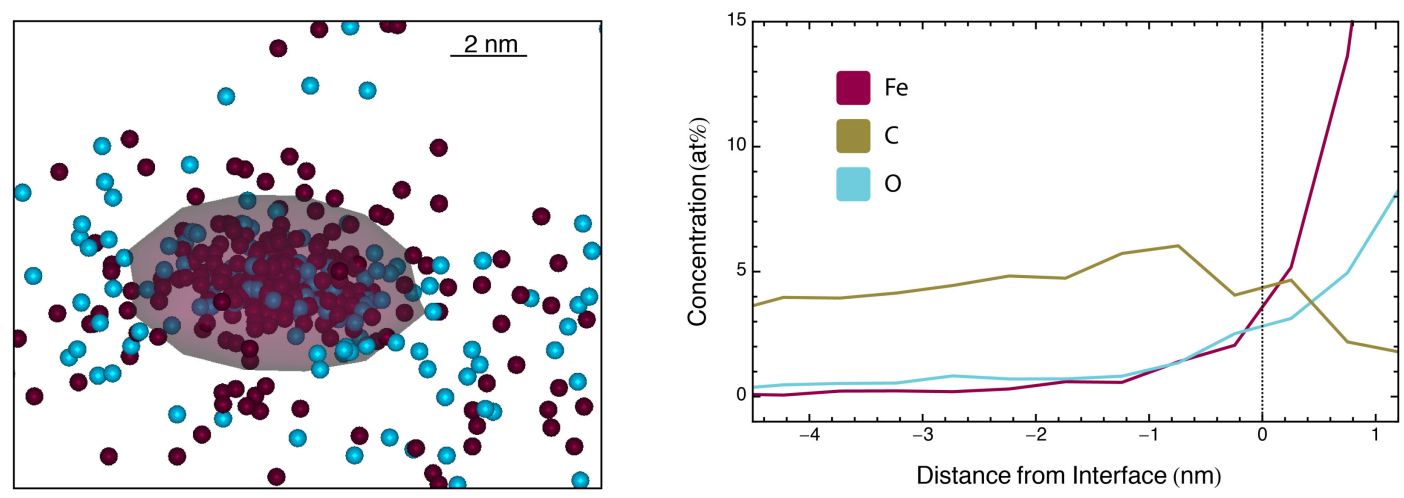

\section{References}

[1] PM Harrison, P Arosio, Biochimica et Biophysica Acta 1275 (1996) p. 161.

[2] ND Chasteen, PM Harrison, J Struct Bio 126 (1999) p. 182.

[3] D. Joester, et al. Microscopy Today. 2012, 20: (2), 26-31

[4] LM Gordon, D Joester, Nature 469 (2011) p. 194.

[5] LM Gordon, L Tran, D Joester, ACS Nano 6 (2012) p. 10667.

[6] ME Greene, TF Kelly et al., J Microscopy 247 (2012) p. 288.

[7] JA Panitz, J Sci Vac Tech 20 (1982) p. 895. 